GASTROINTESTINAL INFECTION

\title{
Clostridium difficile associated diarrhoea in hospitalised patients: onset in the community and hospital and role of flexible sigmoidoscopy
}

\author{
S S Johal, J Hammond, K Solomon, P D James, Y R Mahida
}

Gut 2004;53:673-677. doi: 10.1136/gut.2003.028803

See end of article for authors' affiliations

\section{Correspondence to:} Professor Y R Mahida, Institute of Infection, Immunity, and Inflammation, C Floor, West Block, Queen's Medical Centre

Nottingham NG7 2UH, UK; Yash.Mahida@ Nottingham.ac.uk

Accepted for publication 30 November 2003

\begin{abstract}
Objectives: Clostridium difficile associated diarrhoea (CDAD) is a hospital acquired infection in which optimal methods for diagnosis and the scale of the problem in the community remain to be determined. In hospitalised patients with CDAD, we aimed to (i) study patients in whom the onset of diarrhoea was in the community and (ii) investigate the role of bedside flexible sigmoidoscopy in diagnosis.

Methods: Patients with CDAD (onset in hospital or community) were studied prospectively. In those with diarrhoea of unknown aetiology, flexible sigmoidoscopy was compared with stool assay for $C$ difficile cytotoxin.

Results: Of 136 patients with CDAD (which was associated with antibiotic exposure in 96\%), diarrhoea started in the community in 38 (28\%; majority in own home) and while an inpatient in $98(72 \%)$. The majority with CDAD onset in the community had been hospitalised over the preceding 12 months $(86.8 \% \mathrm{v}$ $57.1 \%$ in the hospital onset group; $\mathrm{p}<0.001)$. In 56 patients with pseudomembranous colitis at sigmoidoscopy, the stool $C$ difficile cytotoxin test was negative in $29(52 \%)$ but toxigenic $C$ difficile was isolated from all of nine stool samples cultured. Of patients with pseudomembranous colitis, $30.4 \%$ relapsed over the subsequent 57.7(4.2) days.

Conclusions: In a significant proportion of hospitalised patients with CDAD, diarrhoea started in the community. However, the majority of these had been hospital inpatients previously when they may have acquired $C$ difficile, with the subsequent onset of diarrhoea in the community following exposure to antibiotics. Flexible sigmoidoscopy is superior to the stool $C$ difficile cytotoxin test in a subgroup of patients with pseudomembranous colitis. Sigmoidoscopy should therefore be considered in all hospitalised patients with diarrhoea in whom the stool test for $C$ difficile cytotoxin and enteric pathogens is negative.
\end{abstract}

lostridium difficile is an anaerobic Gram positive bacillus that is an important cause of diarrhoea in hospitalised patients, which especially affects the elderly. Infection can lead to asymptomatic carriage or clinical disease, ranging from mild diarrhoea to life threatening pseudomembranous colitis. ${ }^{1-3} \mathrm{C}$ difficile associated disease is an important clinical problem that is believed to occur predominantly following hospitalisation and administration of antibiotics. ${ }^{4}$ Moreover, approximately $20 \%$ of patients with Clostridium difficile associated diarrhoea (CDAD) relapse despite initial adequate treatment. ${ }^{5-7}$

CDAD is usually diagnosed following a stool test for $C$ difficile cytotoxin or for the presence of toxigenic $C$ difficile in a cultured stool sample. ${ }^{8}$ As patients may be colonised by nontoxigenic strains of $C$ difficile, any cultured isolates of the bacterium have to be tested in vitro for the production of cytotoxin. Although enzyme linked immunosorbent assays (ELISAs) are increasingly used, demonstration of the presence of $C$ difficile cytotoxin by the characteristic cytopathic effect on cell culture monolayers is considered by many to be the "gold standard". ${ }^{9-11}$ However, the sensitivities of the cell cytotoxicity assays and ELISAs have been reported to be variable. ${ }^{12}{ }^{13}$ In our retrospective audit of 110 patients with $\mathrm{CDAD}$, the first stool sample after the onset of diarrhoea was negative for $C$ difficile cytotoxin in $17 \%$, resulting in a mean delay of 8.2 days before a diagnosis was made and appropriate treatment initiated. ${ }^{14}$ Endoscopic examination of the colon can reveal characteristic yellowish pseudomembranes, often with intervening normal looking mucosa. Although endoscopic examination is recognised to allow rapid diagnosis of $C$ difficile associated pseudomembranous colitis, ${ }^{11}$ its role in the management of patients suspected of having CDAD remains to be determined. Such a role could include establishment of the diagnosis in patients with a false negative stool test for cytotoxin. Diarrhoea in some patients with CDAD resolves without any specific treatment (apart from withdrawal of offending antibiotics) and the role of endoscopic examination in the selection of such patients remains to be determined. It is also unknown whether pseudomembranous colitis predisposes to relapsing disease. We have therefore undertaken a prospective study in which the role of flexible sigmoidoscopy in the diagnosis and management of CDAD was investigated.

CDAD is generally considered to be a disease that is acquired by patients following admission to hospital and administration of antibiotics for another condition (for example, pneumonia). However, CDAD can also be acquired in the community. ${ }^{15-17}$ Of the hospital inpatients with CDAD, the proportion that developed the disease in the community has also been investigated in this prospective study.

\section{METHODS \\ Patients}

Patients that developed diarrhoea ( $\geqslant 3$ watery bowel motions for more than 24 hours) as inpatients or those that were admitted to hospital with diarrhoea from the community were studied. CDAD was diagnosed if: (1) diarrhoea occurred in the presence of $C$ difficile cytotoxin in a stool sample or (2) pseudomembranes were identified at sigmoidoscopy and the

Abbreviations: CDAD, Clostridium difficile associated diarrhoea; ELISA, enzyme linked immunosorbent assay 
diarrhoea responded to oral vancomycin or metronidazole. Suitable patients for this study were identified from daily (during weekdays) visits to the medical (including Health Care of the Elderly) and surgical wards or following referral by medical or nursing colleagues. Those in whom the stool $C$ difficile cytotoxin test was positive by the time the patient was seen by us, had their clinical progress prospectively monitored but did enter the sigmoidoscopy arm of the study. Patients with an unidentified cause for their diarrhoea (who during the same admission may or may not have had a negative stool test for $C$ difficile cytotoxin) were recruited to the sigmoidoscopy arm of the study in which, following informed consent, a bedside flexible sigmoidoscopy was performed and a stool sample was taken at the same time for routine $C$ difficile cytotoxin assay and culture for conventional enteric pathogens (see below).

In addition to demographic data, the following information was collected: onset and severity of diarrhoea, drug treatment, normal residence, and previous hospital admissions. If pseudomembranes were seen at sigmoidoscopy, treatment was started with oral metronidazole or vancomycin. All patients were seen daily until resolution of diarrhoea. This study was approved by the ethics committee of University Hospital NHS Trust.

\section{Flexible sigmoidoscopy}

This examination was undertaken by the bedside using a fibreoptic sigmoidoscope (Olympus OSF-3; KeyMed Ltd, Southend-on-Sea, UK). No bowel preparation was undertaken and if views of the mucosa were obscured by overlying stool, 10-20 $\mathrm{ml}$ of saline was applied down the sigmoidoscope. Unless contraindicated, biopsies were also taken for routine histological examination.

\section{Stool samples}

A stool sample was collected either prior to or at sigmoidoscopy and sent to the clinical enteric laboratory for $C$ difficile cytotoxicity assay (using Vero cells) and culture for conventional enteric pathogens (Salmonella sp, Campylobacter sp, Shigella sp, and Escherichia coli O157) and examination by microscopy for Cryptosporidium. In some, an aliquot of the stool sample was frozen for anaerobic culture at a later date.

For culture studies, stool samples were partially defrosted and $200 \mu \mathrm{l}$ of resultant liquid transferred to an Eppendorf. Samples were then incubated at room temperature for one hour in the presence of an equal volume of $70 \%$ ethanol to remove viable bacteria and select for the presence of spores.

Samples were then streak plated onto $C$ difficile selective media (Oxoid, Basingstoke, Hants, UK) and incubated anaerobically at $37^{\circ} \mathrm{C}$ for two days. $\mathrm{C}$ difficile colonies were identified by distinct colony morphology and by fluorescence under UV light. Toxigenic $C$ difficile were identified by their ability to produce toxin A. Single colonies from each sample were incubated anaerobically in brain heart infusion broth (Oxoid) at $37^{\circ} \mathrm{C}$ for two days. Following centrifugation (at
$2000 \mathrm{~g}$ ), the supernatant was tested for the presence of toxin A using $C$ difficile toxin A test (Oxoid).

\section{Statistics}

Analysis was performed using Fisher's exact test. Results are expressed as mean (SEM).

\section{RESULTS}

Comparison between community versus hospital onset of CDAD

Over a period of 30 months (starting March 2000), 136 patients with CDAD were studied. Of these, diarrhoea developed in the community in $38(28 \%)$, and in the remaining $98(72 \%)$ patients diarrhoea developed in hospital (onset 48 hours after admission for an unrelated condition). Details of the two groups of patients are given in table 1 .

Of patients admitted with diarrhoea, 33 (86.8\%) had been hospitalised over the preceding 12 months compared with 56 $(57.1 \%)$ that developed diarrhoea as inpatients $(p<0.001)$. Patients who developed diarrhoea in the community also had a higher daily stool frequency $(7.2(0.8) v 4.6(0.2) ; \mathrm{p}<0.001)$ and shorter period of hospitalisation (22.6 (3.3) $v 48.9$ (4.0) days; $\mathrm{p}<0.001$ ) but there was no difference in age, duration of diarrhoea, $\mathrm{C}$ reactive protein level, or total white blood count. Of the 38 patients who developed diarrhoea in the community, 21 (55.3\%) were admitted from their own home, eight were from a warden aided complex, five from a residential home, and four from a nursing home. Of these 38 patients, $23(60.5 \%)$ were discharged to their own homes following resolution of the diarrhoea. For patients that developed CDAD in hospital, 68 (69.4\%) were admitted from their own homes but only $30(30.6 \%)$ subsequently returned there $(p<0.001$ compared with those with community onset of CDAD).

The majority of the 136 (96\%) patients had been exposed to antibiotics before developing CDAD, with 114 (84\%) taking cephalosporins and/or broad spectrum penicillins (table 2). Metronidazole, which is often used for the treatment of CDAD, had been taken by $46(33.8 \%)$ patients, in most cases in combination with other antibiotics (table 2).

\section{Investigation of the role of flexible sigmoidoscopy in diagnosis of CDAD}

A subgroup of patients with $\operatorname{CDAD}(n=63)$ took part in a study in which the role of flexible sigmoidoscopy in establishing the cause of diarrhoea was investigated (fig 1). For this arm of the study, 179 hospitalised patients in whom the cause of diarrhoea (onset in community or hospital) was unknown, consented to a study in which findings at flexible sigmoidoscopy were compared with assay for $C$ difficile cytotoxin in a stool sample collected at the same time. Sixty three $(35.2 \%)$ of 179 participating patients were considered to have CDAD based on either the presence of $C$ difficile cytotoxin in the stool sample or the appearance of pseudomembranous colitis at flexible sigmoidoscopy and

Table 1 Clostridium difficile associated diarrhoea (CDAD) onset in the community compared with onset in hospital

\begin{tabular}{|c|c|c|c|c|c|c|c|c|c|}
\hline $\begin{array}{l}\text { Onset of } \\
\text { diarrhoea }\end{array}$ & Age $(y)$ & $\begin{array}{l}\text { Duration of } \\
\text { diarrhoea } \\
\text { (days) }\end{array}$ & $\begin{array}{l}\text { Daily stool } \\
\text { frequency }\end{array}$ & $\begin{array}{l}\text { Patients admitted to } \\
\text { hospital over } \\
\text { preceding } \\
12 \text { months }\end{array}$ & CRP (mg/l) & $\begin{array}{l}\text { WBC } \\
\left(\times 10^{9} / 1\right)\end{array}$ & $\begin{array}{l}\text { Stay time } \\
\text { (days) }\end{array}$ & $\begin{array}{l}\text { Admitted from } \\
\text { own home }\end{array}$ & $\begin{array}{l}\text { Discharged to own } \\
\text { home }\end{array}$ \\
\hline $\begin{array}{l}\text { Community } \\
(n=38)\end{array}$ & 77 (3) & $15(1.8)$ & $7.2(0.8)^{* *}$ & $33(86.8 \%)^{* *}$ & $117(12)$ & $10(0.9)$ & $22.6(3.3)^{\star \star}$ & $21(55.3 \%)$ & $23(60.5 \%)^{* *}$ \\
\hline $\begin{array}{l}\text { As inpatient } \\
(\mathrm{n}=98)\end{array}$ & $79(1)$ & $14(1.3)$ & $4.6(0.2)$ ** & $56(57.1 \%)^{* *}$ & $110(8)$ & $13(0.7)$ & $48.9(4.0)^{* *}$ & $68(69.4 \%)$ & $30(30.6 \%)^{* *}$ \\
\hline
\end{tabular}

Patients developing CDAD in the community had a significantly higher daily stool frequency, shorter duration of stay, more hospital admissions over the preceding 12 months, and were more likely to be discharged to their own home than patients that developed diarrhoea as inpatients (** $p<0.01$ ).

CRP, C reactive protein; WBC, white blood cells. 
Table 2 Intake of antibiotics in Clostridium difficile associated diarrhoea (CDAD) patients

\begin{tabular}{lll}
\hline & $\begin{array}{l}\text { CDAD onset in community } \\
\text { (n=38) }\end{array}$ & $\begin{array}{l}\text { CDAD onset as inpatient } \\
\text { (n=98) }\end{array}$ \\
\hline $\begin{array}{l}\text { Cephalosporin and/or broad spectrum penicillins } \\
\text { prior to onset of diarrhoea }\end{array}$ & $31(81.6 \%)$ & $83(84.7 \%)$ \\
$\begin{array}{l}\text { Metronidazole in addition to cephalosporin and/or } \\
\text { broad spectrum penicillins }\end{array}$ & $12(31.2 \%)$ & $23(23.5 \%)$ \\
$\begin{array}{l}\text { Patients not on cephalosporins or penicillins } \\
\text { Metronidazole }\end{array}$ & $7(18.4 \%)$ & $15(15.3 \%)$ \\
Ciprofloxacin & $1(2.6 \%)$ & $1(1 \%)$ \\
Ciprofloxacin (with metronidazole) & $1(2.6 \%)$ & $2(2 \%)$ \\
Erythromycin (with metronidazole) & $1(2.6 \%)$ & $3(3.1 \%)$ \\
Trimethoprim (1 with ciprofloxacin) & $1(2.6 \%)$ & $3(3.1 \%)$ \\
Erythromycin+ciprofloxacin (with metronidazole) & $0(0 \%)$ & $13.1 \%)$ \\
No antibiotics & $0(0 \%)$ & $2(2 \%)$ \\
Patients on $<3$ antibiotics & $3(7.9 \%)$ & $75(53.1 \%)$ \\
Patients on $\geqslant 3$ antibiotics & $25(65.8 \%)$ & $46(46.9 \%)$ \\
\hline Values are number (\%). & $13(34.2 \%)$ & \\
\hline
\end{tabular}

response to metronidazole and/or vancomycin. Duration of diarrhoea in the CDAD patients prior to sigmoidoscopy was 10.1 (1.2) days. The causes of diarrhoea in the 116 patients without CDAD are given in table 3.

In 56 patients with CDAD (88.9\%), flexible sigmoidoscopy showed pseudomembranous colitis (examinations limited to the rectum/distal sigmoid colon), with rectal sparing (pseudomembranes limited to the sigmoid colon) in six $(10.7 \%)$. Mucosal biopsies from patients with a sigmoidoscopic appearance of pseudomembranous colitis showed histological changes characteristic of pseudomembranous colitis in 40 patients and colonic inflammation in 15 patients (one patient was on warfarin and therefore biopsies were not taken). However, $C$ difficile cytotoxin assay in a stool sample collected at the same time as flexible sigmoidoscopy was negative in 29 patients (52\% of patients with pseudomembranous colitis) (fig 1). Diarrhoea in all of these latter patients resolved on treatment with oral metronidazole and/or vancomycin over a period of 7.8 (1.5) days (compared with 7.0 (1.3) days in

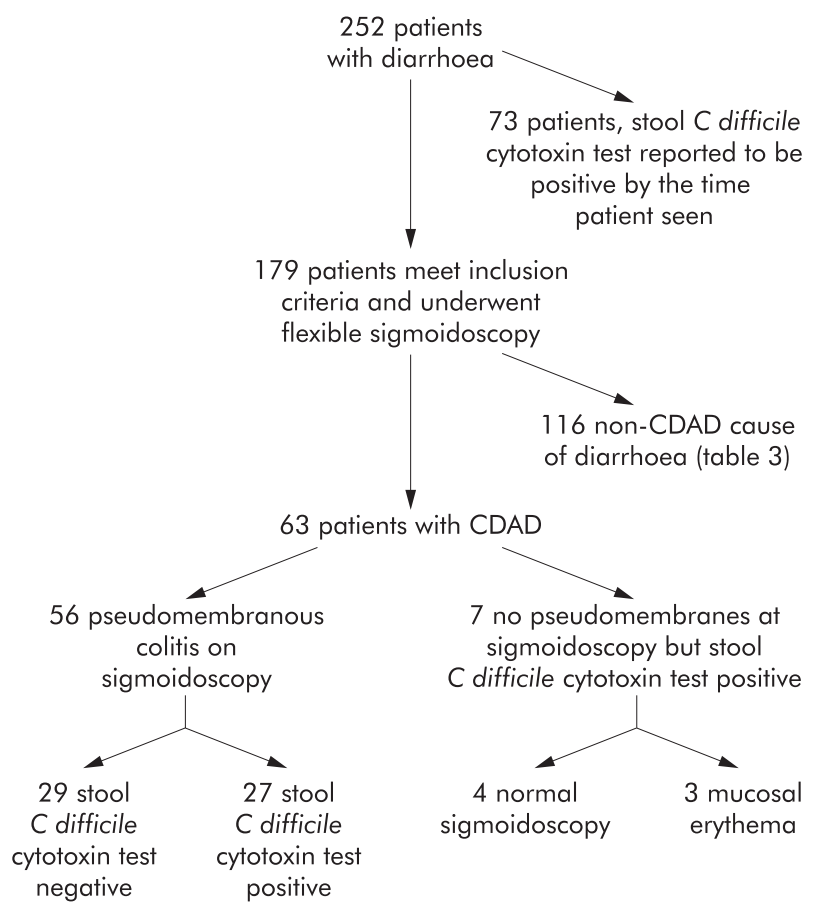

Figure 1 Outcome in patients that underwent flexible sigmoidoscopy. CDAD, Clostridium difficile associated diarrhoea. those with pseudomembranous colitis and a positive stool test for $C$ difficile cytotoxin). Sixteen of 29 pseudomembranous colitis patients with a negative stool test for $C$ difficile cytotoxin also had a negative stool test for cytotoxin prior to sigmoidoscopy (sample taken during the same episode of diarrhoea; mean 12.4 (3.9) days before sigmoidoscopy). None of the patients experienced any adverse effects as a result of flexible sigmoidoscopy.

Frozen aliquots of nine stool samples that were negative for $C$ difficile cytotoxin (taken at the time of flexible sigmoidoscopy) were available for anaerobic culture and all revealed colonies of organisms with typical characteristics (morphology and fluorescence under UV light) of $C$ difficile. These organisms were confirmed to be toxigenic $C$ difficile by demonstration of the presence of toxin A in supernatant samples of subcultured colonies. In four patients with a negative stool $C$ difficile cytotoxin test at the time of flexible sigmoidoscopy, subsequent stool samples (taken following initiation of treatment) were positive for $C$ difficile cytotoxin (in two of these patients, anaerobic culture of an aliquot of stool sample collected at the time of flexible sigmoidoscopy grew toxigenic $C$ difficile).

Fifty four of 56 (96.4\%) patients with pseudomembranous colitis were treated with oral metronidazole or vancomycin, with resolution of diarrhoea over 8.4 (1.0) days. In two patients with mild diarrhoea and early pseudomembranes, the bowel habit returned to normal on discontinuation of the offending antibiotics. In seven of 63 CDAD patients without

Table 3 Causes of diarrhoea, other than Clostridium difficile associated diarrhoea, in patients that underwent flexible sigmoidoscopy

\begin{tabular}{ll}
\hline Cause of diarrhoea & No of patients \\
\hline Antibiotics & 22 \\
Probable gastrointestinal infection & 19 \\
Unknown cause & 17 \\
Constipation with overflow & 13 \\
Campylobacter infection & 11 \\
Inflammatory bowel disease & 11 \\
Salmonella infection & 5 \\
Ischaemic colitis & 4 \\
Irritable bowel syndrome & 4 \\
Carcinoid & 3 \\
Drug induced & 2 \\
Coeliac disease & 1 \\
Pancreatic insufficiency & 1 \\
Collagenous colitis & 1 \\
E coli 0157 & 1 \\
Colonic cancer & 1 \\
\hline
\end{tabular}


pseudomembranous colitis, diarrhoea resolved within 24 hours of discontinuation of the offending antibiotics in four $(\mathrm{p}<0.001$ compared with patients with pseudomembranous colitis) and the remaining three patients responded to treatment with metronidazole and/or vancomycin.

\section{Stool C difficile cytotoxicity test and treatment in patients not sigmoidoscoped}

Of 73 patients with CDAD that did not take part in the sigmoidoscopy study, 26 (36\%) had an initial negative stool test prior to the subsequent sample that established the diagnosis. Mean delay before sending the second stool sample was 13.1 (2.3) days. Fifty one (69.6\%) patients in this group were treated with metronidazole and/or vancomycin with resolution of diarrhoea over 8.7 (1.0) days. In the remaining $22(30.1 \%)$ patients, diarrhoea resolved (over 4.0 (0.7) days) after discontinuation of the offending antibiotics.

\section{Relapse of CDAD}

Seventeen $(30.4 \%)$ of 56 patients with pseudomembranous colitis at sigmoidoscopy relapsed over the subsequent 57.7 (4.2) days. None of the seven patients without pseudomembranous colitis relapsed. In total, there were 20 CDAD relapse episodes, with 14 patients relapsing once and three patients relapsing twice. Broad spectrum antibiotics were taken during nine relapse episodes but there was no such history in the rest. Flexible sigmoidoscopy performed during 16 relapse episodes showed pseudomembranous colitis in 14 $(87.5 \%)$. Of the stool samples sent during 19 relapse episodes, six $(32 \%)$ were negative for $C$ difficile cytotoxin. Frozen aliquots of five stool samples that were negative for $C$ difficile cytotoxin were cultured anaerobically, and colonies of toxigenic $C$ difficile (confirmed by the presence of toxin $\mathrm{A}$ in supernatant samples of subcultures) were identified in four.

Of 73 patients with CDAD that were not in the sigmoidoscopy study, $15(21 \%)$ relapsed over the subsequent 42.1 (8.0) days.

\section{DISCUSSION}

CDAD is an infection of great clinical importance in hospitalised patients the incidence of which has increased markedly over the past 10 years $^{4}$ (www.hpa.org.uk). In our hospital, the number of patients with $C$ difficile infection increased from 29 in 1993 to 210 in 1995. A retrospective analysis of the case notes of 110 patients in 1995 showed that the first stool test for $C$ difficile cytotoxin was negative in a significant minority of patients (17\%), leading to a significant delay (8.2 (6.6) days) before the second diagnostic stool sample allowed appropriate treatment and infection control measures to be undertaken. ${ }^{14}$ It is well recognised that the sensitivity of neither the stool cytotoxicity test nor the ELISA tests is $100 \% .^{12}{ }^{13} 18$ As endoscopic examination can reveal characteristic appearances of pseudomembranous colitis, our aim was to prospectively investigate the role of flexible sigmoidoscopy, at the bedside, in the diagnosis and management of CDAD. Flexible, rather than rigid, sigmoidoscopy was used because rectal sparing can occur in some patients with pseudomembranous colitis ${ }^{19}$ (which was confirmed in $10.7 \%$ of patients in our study). In order to determine the role of flexible sigmoidoscopy, it was felt that a "head to head" comparison with the stool cytotoxin test would be appropriate because, to our knowledge, such a prospective study has not been previously performed. Of a total of 136 patients with CDAD, in $73(53.7 \%)$ the diagnosis had already been made (and treatment initiated) following a positive stool test for $C$ difficile cytotoxin (prior to our seeing the patient). Review of the notes and results of these patients showed that in $36 \%$, the first stool test for C difficile cytotoxin had been negative and the diagnosis of CDAD was not established until a second stool sample (sent by the team looking after the patient) was sent a mean 13.1 (2.3) days after the first. Following diagnosis of CDAD, these patients were subsequently followed up by us until resolution of diarrhoea.

A total of 179 patients with persistent diarrhoea of unknown cause entered that part of the study in which flexible sigmoidoscopy was compared with the stool $C$ difficile cytotoxin test. Sixty three patients in this group were considered to have CDAD, with flexible sigmoidoscopy showing pseudomembranes in 56. In those with pseudomembranous colitis, the stool $C$ difficile cytotoxin test (performed at the same time as sigmoidoscopy) was negative in $29(52 \%)$. A previous stool $C$ difficile cytotoxin test was also negative in a significant proportion of the latter group (16; $55.2 \%$ of those in whom the stool $C$ difficile cytotoxin test was negative at the time of sigmoidoscopy). This raises the possibility that pseudomembranous colitis in these patients was due to another enteric pathogen and not toxigenic $C$ difficile. ${ }^{20}$ We believe that pseudomembranous colitis in our patients was due to toxigenic $C$ difficile for the following reasons. All of these patients responded to oral vancomycin and/or metronidazole over a period of time that was similar to those with pseudomembranous colitis and a positive $C$ difficile cytotoxin test. Moreover, in nine patients (with a negative stool $C$ difficile cytotoxin test) in whom a frozen aliquot of stool sample (taken at the same time as sigmoidoscopy) was available, toxigenic $C$ difficile was isolated during anaerobic culture. Enterotoxigenic $C$ perfringens can cause antibiotic associated diarrhoea (which can respond to metronidazole) but to our knowledge this infection has not been reported to cause pseudomembranous colitis. ${ }^{21} 22$

The reasons for negative stool $C$ difficile cytotoxin tests in many of the CDAD patients in our study are unknown. For the sigmoidoscopy arm of the study, stool samples were collected and sent to the enteric laboratory on the same day as the endoscopic examination. Although there was no delay in sending these stool samples, it is conceivable that toxin degradation may occur rapidly in some samples because of the presence of proteases derived from other bacteria. Another possibility is that other components in stool samples (which may also be derived from bacteria) may interfere with the bioassay by binding to the toxins. Further studies are required to address these possibilities.

As stool $C$ difficile cytotoxicity assays and ELISAs for toxins $\mathrm{A}$ and $\mathrm{B}$ are known to be negative in a proportion of patients with CDAD, it has been suggested that either stool culture for toxigenic $C$ difficile and toxin detection should be performed at the same time on all stool specimens ${ }^{23}$ or toxigenic culture performed on those with a negative stool toxin assay. ${ }^{18}$ The former strategy would allow more cases of CDAD to be diagnosed but would impose a significant burden on the laboratory services, particularly as cultured $C$ difficile would have to be confirmed to be toxigenic (by demonstration of toxin secretion or the presence of their genes), and a positive result would not be a significant addition in those patients with a positive stool toxin assay. Toxigenic culture in patients with a negative stool toxin assay would require either an aliquot of the original stool sample to be stored or another sample collected for anaerobic culture. Moreover, anaerobic culture and identification of toxigenic $C$ difficile would take some time, during which the patient may deteriorate and continue to pose a risk of transmission of infection to neighbouring patients. Our study has shown that flexible sigmoidoscopy is superior to stool $C$ difficile cytotoxin test in a subgroup of patients with $C$ difficile associated pseudomembranous colitis. Because of the characteristic appearance of pseudomembranous colitis, flexible sigmoidoscopy would allow a rapid diagnosis in patients with a negative stool toxin assay, enabling treatment and infection control 
measures to be initiated, particularly as transmission of infection has been shown to occur in hospital. ${ }^{24-26}$ Endoscopy would also allow an assessment of the severity of the colitis in these patients, thereby facilitating subsequent management.

As shown in table 3, many of the hospitalised patients with persistent diarrhoea and a negative $C$ difficile toxin assay will not have CDAD but other conditions such as ulcerative colitis, Crohn's disease, ischaemic colitis, or infectious enterocolitis. As the endoscopic appearances in many of these conditions are characteristic, together with the facility to take biopsies for histology and stool samples for further microbiological studies, flexible sigmoidoscopy could be of considerable benefit in the management of hospitalised patients with diarrhoea. However, because it is non-invasive and more cost effective, we agree with the recommendation that examination of a stool sample for $C$ difficile toxins should be the initial investigation of choice in a hospitalised patient with diarrhoea. ${ }^{27}$ In the presence of continuing diarrhoea and a negative stool test for $C$ difficile toxins (and other enteric pathogens), we recommend flexible sigmoidoscopy as it will enable a rapid diagnosis in those patients with pseudomembranous colitis who need treatment the most. Our study has shown that flexible sigmoidoscopy is well tolerated in these patients and can be undertaken by the bedside with the advantage of minimising the risk of transmission of the disease by transportation to the endoscopy unit.

Our study suggests that in the absence of pseudomembranes at sigmoidoscopy, specific treatment (apart from discontinuation of the offending antibiotics) may not be necessary but further studies are required to determine whether endoscopic findings are superior to clinical features in making such decisions about management. As none of the small number of CDAD patients without pseudomembranes at sigmoidoscopy relapsed during the study, it is possible that pseudomembranous colitis increases the risk of subsequent relapse. However, the difference in our study was not statistically significant and further work is required. If the presence of pseudomembranes is shown to be associated with the risk of relapse, such patients could be selected for studies to investigate therapeutic agents that may reduce this risk.

A significant proportion (28\%) of our patients with CDAD developed their disease in the community (in their own homes in over half of these patients) following administration of antibiotics. The majority of these patients had been hospital inpatients over the preceding 12 months, raising the possibility that colonisation with toxigenic $C$ difficile, without significant symptoms, occurred during such an admission. Asymptomatic acquisition of toxigenic $C$ difficile following admission to hospital has been reported..$^{24}$ If this were the case, growth of toxigenic $C$ difficile could occur following antibiotic induced disruption of the normal bowel flora and would suggest the cautious use of antibiotics in community elderly patients who have had recent hospital admissions.

\section{ACKNOWLEDGEMENTS}

This study was supported by the Community Fund (formerly National Lottery Charities Board) via the Digestive Disorders Foundation. We thank Dr Vivienne Weston for helpful comments.

\author{
Authors' affiliations \\ S S Johal, J Hammond, Division of Gastroenterology, University of \\ Nottingham, Nottingham, UK \\ K Solomon, Y R Mahida, Division of Gastroenterology, and Institute of
}

Infection, Immunity, and Inflammation, University of Nottingham, Nottingham, UK

P D James, Division of Pathology, University of Nottingham, Nottingham, UK

\section{REFERENCES}

1 Larson $\mathrm{HE}$, Price $A B$, Honour $P$, et al. Clostridium difficile and the aetiology of pseudomembranous colitis. Lancet 1978;1:1063-6.

2 Bartlett JG, Tedesco FJ, Shull S, et al. Symptomatic relapse after oral vancomycin therapy of antibiotic-associated pseudomembranous colitis. Gastroenterology 1980;78:431-4.

3 Cleary RK. Clostridium difficile-associated diarrhea and colitis: Clinical manifestations, diagnosis, and treatment. Dis Colon Rectum 1998;41:1435-49.

4 Wilcox $\mathrm{MH}$. Cleaning up Clostridium difficile infection. Lancet 1996;348:767-8.

5 Fekety R, McFarland LV, Surawicz CM, et al. Recurrent Clostridium difficile diarrhea: Characteristics of and risk factors for patients enrolled in a prospective, randomized, double-blinded trial. Clin Infect Dis, 1997;24:324-33.

6 Walters BA, Roberts R, Stafford R, et al. Relapse of antibiotic associated colitis: endogenous persistence of Clostridium difficile during vancomycin therapy. Gut 1983;24:206-12.

7 Bartlett JG, Chang TW, Gurwith M, et al. Antibiotic-associated pseudomembranous colitis due to toxin-producing clostridia. N Engl J Med 1978;298:531-4.

8 Brazier JS. The epidemiology and typing of Clostridium difficile. J Antimicrob Chemother 1998;41(suppl C):47-57.

9 Barbut F, Kajzer C, Planas N, et al. Comparison of three enzyme immunoassays, a cytotoxicity assay, and toxigenic culture for diagnosis of Clostridium difficile-associated diarrhea. J Clin Microbiol 1993;31:963-7.

10 Bond F, Payne G, Borriello SP, et al. Usefulness of culture in the diagnosis of Clostridium difficile infection. Eur J Clin Microbiol Infect Dis, 1995;14:223-6.

11 Fekety R. Guidelines for the diagnosis and management of Clostridium difficile-associated diarrhea and colitis. American College of Gastroenterology, Practice Parameters Committee. Am J Gastroenterol 1997;92:739-50.

12 Manabe YC, Vinetz JM, Moore RD, et al. Clostridium difficile colitis: an efficient clinical approach to diagnosis. Ann Intern Med 1995; 123:835-40.

13 Gerding DN. Diagnosis of Clostridium difficile-associated disease: patient selection and test perfection. Am J Med, 1996;100:485-6.

14 Wight N, Curtis H, Hyde J, et al. Clostridium difficile-associated diarrhoea. Postgrad Med J, 1998;74:677-8.

15 Hirschhorn LR, Trnka Y, Onderdonk A, et al. Epidemiology of communityacquired Clostridium difficile-associated diarrhea. J Infect Dis, 1994; 169:127-33.

16 Kyne L, Merry C, O'Connell B, et al. Community-acquired Clostridium difficile infection. J Infect 1998;36:287-8.

17 Levy DG, Stergachis A, McFarland LV, et al. Antibiotics and Clostridium difficile diarrhea in the ambulatory care setting. Clin Ther 2000;22:91-102.

18 Lozniewski A, Rabaud C, Dotto E, et al. Laboratory diagnosis of Clostridium difficile-associated diarrhea and colitis: usefulness of Premier Cytoclone $A+B$ enzyme immunoassay for combined detection of stool toxins and toxigenic $\mathrm{C}$. difficile strains. J Clin Microbiol, 2001;39:1996-8.

19 Tedesco FJ, Corless JK, Brownstein RE. Rectal sparing in antibiotic-associated pseudomembranous colitis: a prospective study. Gastroenterology 1982:83:1259-60.

20 Phillips RK, Glazer G, Borriello SP. Non-Clostridium difficile pseudomembranous colitis responding to both vancomycin and metronidazole. Br Med J Clin Res Ed 1981;283:823.

21 Borriello SP, Welch AR, Larson HE, et al. Diarrhoea and simultaneous excretion of Clostridium difficile cytotoxin and $C$ perfringens enterotoxin. Lancet 1984;2:1218.

22 Asha NJ, Wilcox MH. Laboratory diagnosis of Clostridium perfringens antibiotic-associated diarrhoea. J Med Microbiol, 2002;51:891-4.

23 Delmee M. Laboratory diagnosis of Clostridium difficile disease. Clin Microbiol Infect 2001;7:411-16.

24 McFarland LV, Mulligan ME, Kwok RY, et al. Nosocomial acquisition of Clostridium difficile infection. N Engl J Med, 1989;320:204-10.

25 Clabots CR, Johnson S, Olson MM, et al. Acquisition of Clostridium difficile by hospitalized patients: evidence for colonized new admissions as a source of infection. J Infect Dis, 1992;166:561-7.

26 Samore MH, Venkataraman L, DeGirolami PC, et al. Clinical and molecular epidemiology of sporadic and clustered cases of nosocomial Clostridium difficile diarrhea. Am J Med, 1996;100:32-40.

27 Fekety R. Guidelines for the diagnosis and management of Clostridium difficile-associated diarrhea and colitis. American College of Gastroenterology, Practice Parameters Committee. Am J Gastroenterol 1997;92:739-50.

28 Kyne L, Warny M, Qamar A, et al. Asymptomatic carriage of Clostridium difficile and serum levels of lgG antibody against toxin A. N Engl J Med 2000;342:390-7. 